\title{
Modification of the field enhancement factor for a field emitter with a surrounding electrode stabilized using a field effect transistor
}

\author{
Yoichiro $\mathrm{NeO}^{\mathrm{a})}$ \\ Research Institute of Electronics, Shizuoka University, 3-5-1 Johoku, Hamamatsu, 432-8011, Japan \\ Takahiro Matsumoto \\ Research and Development Center, Stanley Electric Corporation, 5-9-5 Tokodai, Tsukuba 300-2635, Japan \\ Hidetaka Shimawaki \\ Department of System and Information Engineering, Hachinohe Institute of Technology, 88-1 Ohbiraki, \\ Myo, Hachinohe 031-8501, Japan \\ Hidenori Mimura \\ Research Institute of Electronics, Shizuoka University, 3-5-1 Johoku, Hamamatsu, 432-8011, Japan \\ Kuniyoshi Yokoo \\ Ideal Star Inc., 6-6-3, Minamiyoshinari Aobaku, Sendai, 989-3204, Japan
}

(Received 4 September 2007; accepted 28 November 2007; published 1 April 2008)

\begin{abstract}
One of the useful methods for stabilizing field emission current in field emitter arrays (FEAs) is to control them using field effect transistors (FETs) connected in series. However, one cannot use conventional FETs, because the electron extraction voltage (typically hundreds of volts) of FEAs is much higher than the permitted source-drain voltage of FETs. The authors conducted a detailed investigation of the mechanism for current stabilization of the field emission current while controlling the field emitter tip wtih a FET. The electron source consists of the FET controlled tip and surrounding electrode, which is kept at a constant potential. In this paper the authors discuss how the field enhancement factor $(\beta)$ is modified by the potential distribution, which is determined by the potentials of the tip and the surrounding electrode, Making use of this effect, the authors could control a FEA that has a high electron extraction voltage with a FET with a lower source-drain voltage. () 2008 American Vacuum Society. [DOI: 10.1116/1.2827502]
\end{abstract}

\section{INTRODUCTION}

The emission currents from field emitters suffer from step, spike, and flicker noise, even when operated in ultra high vacuum. ${ }^{1}$ Eliminating this noise and suppressing current fluctuations in flat panel displays that use field emitter arrays (FEAs) is extremely desirable. A method for stabilizing the field emission current of a FEA has already been proposed. ${ }^{2,3}$ In this system field effect transistors (FETs) including thin film transistors (TFTs) were connected in series with the FEA. The principle of stabilizing the FEA using FETs can be explained as follows: The FET limits the injection current to the emitter tip during operation. In this situation, the potential of the tip increases. As a result, the source-drain voltage increases and the voltage between the electron extraction electrode and the tip decreases. The tip potential then reaches equilibrium where the emission current is equal to the injection current. At equilibrium, reduction in the work function due to the adsorption of residual gas molecules increases the emission current whereas an increase in the tip potential reduces it. The typical extraction gate voltage for a FEA for electron emission is about $100 \mathrm{~V}$, , which is much bigger than the source-drain voltage of a conventional FET. To support this high voltage, a multi-gate and a lightly doped drain region has been used. ${ }^{5}$ However, the area of this FET was

${ }^{\text {a)} E l e c t r o n i c ~ m a i l: ~ y-n e o @ ~ r i e . s h i z u o k a . a c . j p ~}$ several hundred square micrometers. ${ }^{4}$ This means that flat panel displays with small pixels cannot be realized using FET controlled FEAs.

To overcome this problem, we carried out a detailed investigation of the mechanism for stabilizing the field emission current in FET controlled field emitters. In this paper, we propose a new stabilization system, which consists of a FET connected in series with the tip, and a surrounding electrode kept at a constant potential. In this system, the field enhancement factor $(\beta)$ is modified by the potential distribution, which is in turn determined by the potentials of the tip and the surrounding electrode. With this system only small increases in tip potential are needed to reduce the emission current. That is, the emission current can be suppressed without significantly increasing the source-drain voltage of the FET. Using this effect, even a FET with a low permitted source-drain voltage can be used to control a FEA with a high electron extraction voltage.

\section{EXPERIMENT}

Figure 1 shows the experimental apparatus. All experiments were carried out in an ultra high vacuum chamber, which was pumped below $8.0 \times 10^{-8} \mathrm{~Pa}$. The electron source used in the experiments consisted of a tungsten field emitter tip, made by electrochemically etching a tungsten wire, and a surrounding electrode near the bottom of the tip. The height of the tip above the surrounding electrode was 


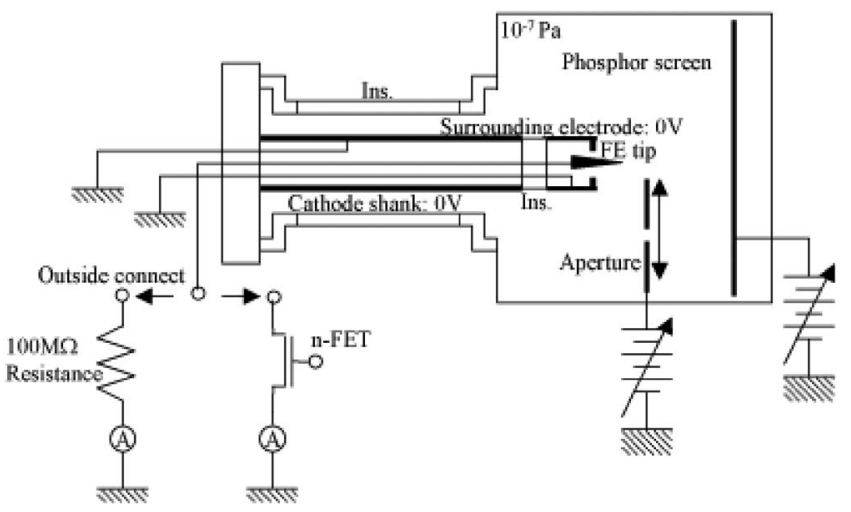

Fig. 1. Measurement apparatus. An $n$-FET or a $100 \mathrm{M} \Omega$ resisitor is connected to the field emitter tip outside the chamber.

several $\mathrm{mm}$. The electron source was set on a cathode shank and located in the center of the chamber. A metal-backed phosphor screen, which functioned as an electron extraction electrode (anode) and was also used for measuring emission currents and emission patterns, was located $5 \mathrm{~cm}$ in front of the tungsten tip. Another extraction electrode with a $2.0 \mathrm{~mm}$ $\phi$ diameter aperture was set on a manipulator and placed in front of the tip. To keep the emission current constant, an $n$-channel field effect transistor (TOSHIBA: 2SK2013) was connected in series with the tip., ${ }^{2,3}$ The impedance between the tip and the extraction electrode was greater than $1 \times 10^{10} \Omega$ during operation. In order to prevent the emission current measured from influencing result, we used a noncontact voltage meter (Kelvin probe, Trek Japan: Model 323). To examine this measurement system, the tip was connected to a $100 \mathrm{M} \Omega$ resistor, as well as the FET. The potential of the tip, depicted by an arrow in Fig. 1, was measured using the Kelvin probe.

\section{RESULTS AND DISCUSSIONS}

Figure 2(a) shows the emission characteristics of a tip connected to a $100 \mathrm{M} \Omega$ resistor. As the extraction voltage increases, both the emission current and the potential on the resistor also increase. Figure 2(b) indicates the emission current and the potential on the resistor as functions of time. The potential divided by the emission current is consistent with the $10^{8} \Omega$ resistor used in this experiment.

Figure 3(a) shows the emission characteristics of the emitter tip controlled by the FET for various FET gate voltages. Current saturation regions occur, where the emission current is independent of the anode-cathode voltage, but depends only on the gate voltage of the FET. Therefore, the emission current has been stabilized in these areas. In stabilized operation, the tip potential, which is at the same voltage $(V d)$ as the drain of the FET, must be shifted by the same amount as the difference in voltage $(\Delta V a)$ between the applied anode voltage and the anode voltage at the onset of stabilized operation. Figure 3(b) shows the increase of $V d$ with increasing $V a$ under stabilized operation for various FET gate voltages $(V g)$. Without FET control, a reduction of $10 \mathrm{nA}$ in the emission current corresponds to a reduction of $100 \mathrm{~V}$ on the an-
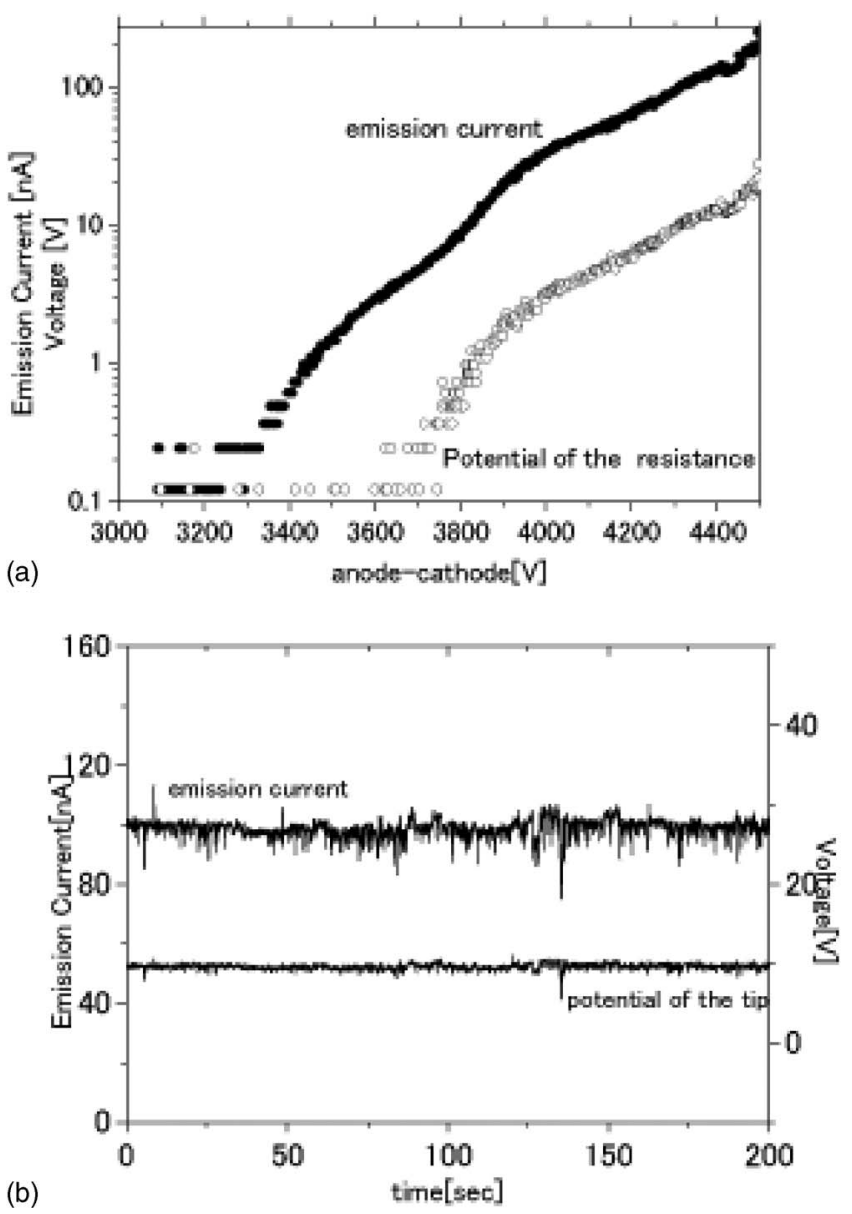

FIG. 2. (a) Emission characteristics when the tungsten tip is connected to the $100 \mathrm{M} \Omega$ resistor. The solid and open circles refer to the emission current and potential on the resistor, respectively. (b) Emission current and potential on the resisitor as a function of time.

ode [refer to the emission currents at 3700 and $3800 \mathrm{~V}$ in Fig. 3(a)]. However, in the system using FET control, reducing $V d$ by less than $30 \mathrm{~V}$ is sufficient for a $10 \mathrm{nA}$ reduction as shown in Fig. 3(b). In our apparatus, the work function and carrier density were identical both with and without stabilized operation. According to the Fowler-Nordheim equation, the emission current under stabilized operation is determined only by $\beta$ and the voltage between the anode and the tip. If $\beta$ is constant, the tip potential should be equal to $\Delta V a$. However, the result shown in Fig. 3 indicates that the emission current is controlled not only by $\Delta V a$ but also $\beta$. The electric field distribution under stabilized operation was investigated using a simulator (SIMION 3D 7.0). Figure 4 shows the electric field around the tip. The saddle point appears near the surrounding electrode toward the tip and reduces the electric field at the end of the tip.

To investigate in detail, modifications of the tip potential by $\beta$ characteristic measurements with a retarding potential were carried out. In the measurements, a certain bias voltage was applied to the gate of the FET, several $\mathrm{kV}$ were applied to the aperture to extract electrons from the tip, and a retarding voltage was applied to the phosphor screen. With $V g$ at $0.6 \mathrm{~V}$ and an emission current of about $1 \mathrm{nA}$ the current was 

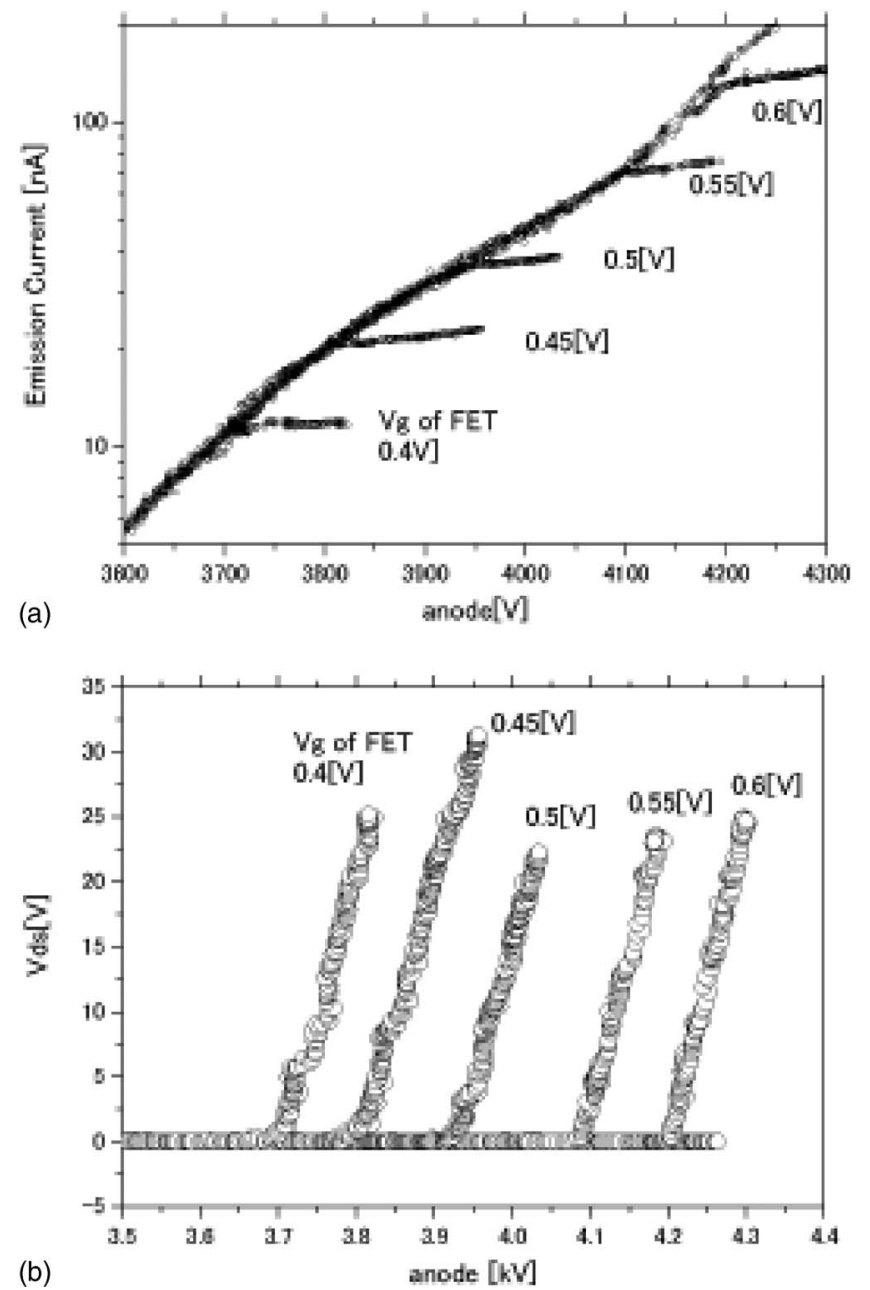

FIG. 3. (a) Emission characteristics of the emitter tip controlled by a FET for various FET-gate voltages. (b) Dependence of $V d$ on anode voltage under stabilized operation for various gate voltages on the FET.

not saturated and $V d$ was equal to zero. By reducing $V g$ from 0.5 to $0.47 \mathrm{~V}$, the emission current was suppressed by the FET and $V d$ increased to $29 \mathrm{~V}$. Figure 5(a) shows the retarding characteristics. The voltage shift increased with decreas-

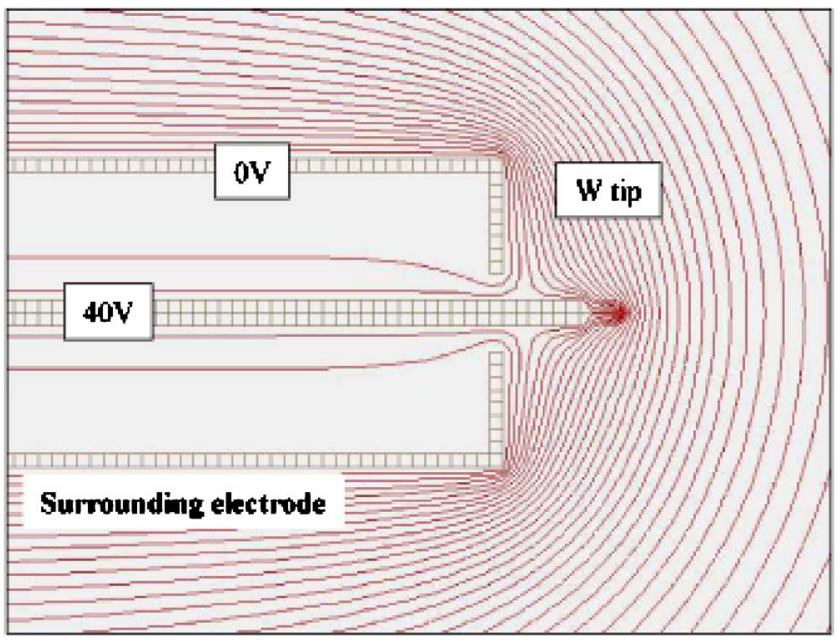

FIG. 4. Electric field distribution under operation at the reduced $\beta$
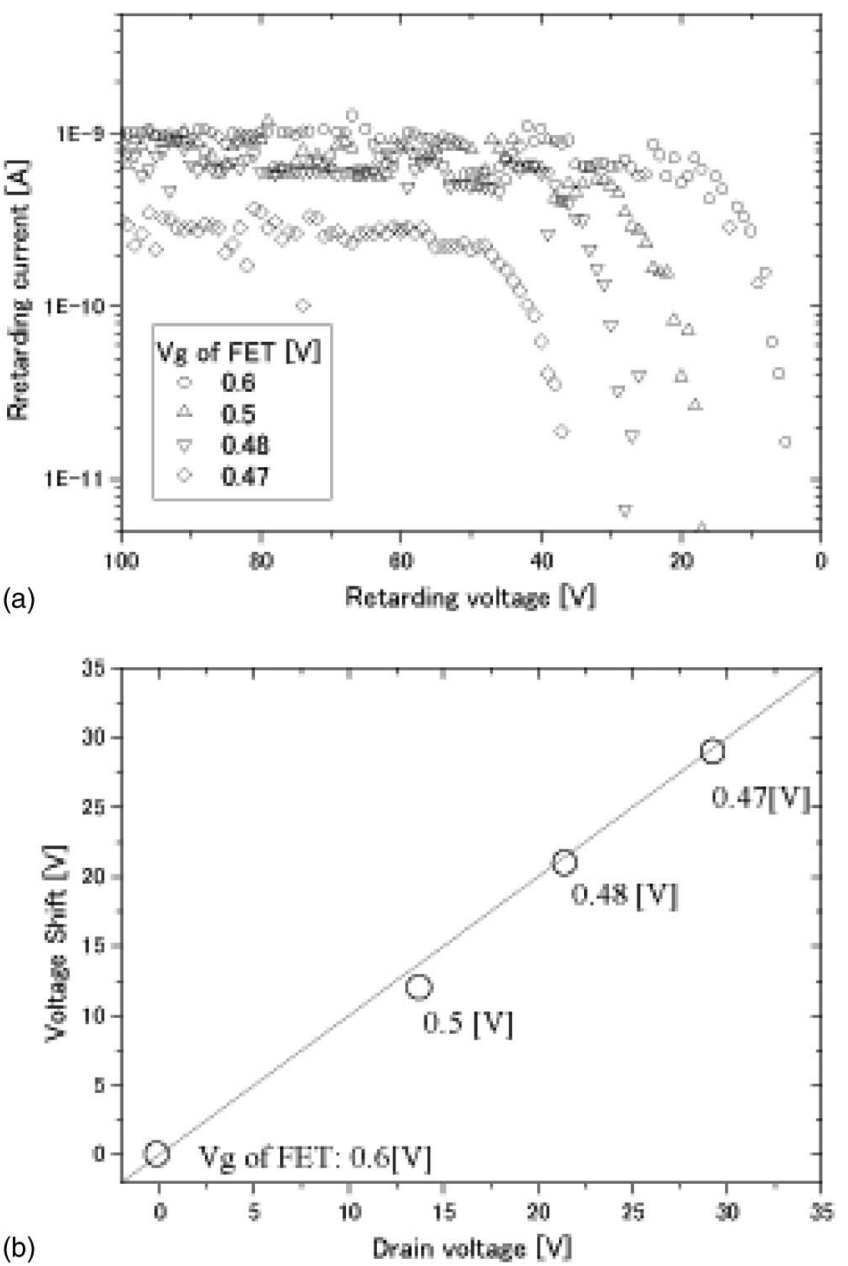

FIG. 5. (a) Retarding characteristics for various gate voltages on the FET. (b) Relationship between the voltage shift of the retarding voltage and the drain voltage.

ing $V g$ bias voltage. The retarding voltages at which the emission current was reduced by $10 \%$ were compared at various gate bias voltages. The relationship between the shift in the retarding voltage and the drain voltage is shown in Fig. 5(b), which shows the voltage shift varies linearly with drain voltage. These results indicate that the tip potential can be measured correctly using this measurement system. The results also suggest that $\beta$ is modified by changes in electric field distribution. $\beta$ decreases during stabilized operation and, as a result, the increase in $V d$ was reduced to about $30 \%$ of $100 \mathrm{~V}$. It is considered that $\beta$ is modified by the surrounding electrode, which is kept at a constant voltage.

To confirm that the modification of $\beta$ is caused by the constant potential of the surrounding electrode, a similar experiment to that shown in Fig. 3 was carried out. In this new experiment, the surrounding electrode and cathode shank were connected to the tip, while in Fig. 3 they were maintained at $0 \mathrm{~V}$. In this condition, $\beta$ was constant and the shift in $V d$ of the FET matched the increase in anode voltage $(\Delta V a)$. In this case, the width of the stabilization region was limited by the maximum permitted source-drain voltage of the FET. 
An electron source, which consists of a tip and a surrounding electrode kept at constant potential, has already been realized in a FEA with vertical-type junction field effect transistor (JFET) ${ }^{6}$ In this device, a constant $p$-type area surrounds an $n$-type tip. The extraction electrode and $p$-type area played the same roles as the anode and the surrounding electrode in our current apparatus. Even if the extraction electrode is near to the tip, the electrical field would be a similar distribution in our apparatus when the potential at the $n$-type tip region is increasing. Part of the electric field, which terminates at the tip without stabilized operation, terminates at the $p$-type area under stabilized conditions. Thus, in the FEA with vertical-type JFET, $\beta$ modification occurred. Taking the fabrication process into consideration, a FEA with vertical-type JFET is a potential candidate for controlling emission by modifying $\beta$.

\section{CONCLUSIONS}

We have carried out a detailed investigation of a mechanism for stabilizing the current from a field emission current from a field emitter tip controlled by a FET. For an electron source consisting of a tip and a surrounding electrode at a constant potential, the shift in the drain voltage of the FET was much less than $\Delta V a$. On the other hand, when the surrounding electrode and cathode shank were connected to the tip, the shift in $V d$ corresponded with $\Delta V a$. This suggests that $\beta$ is modified by the potential distribution, which is determined by the potential of the tip controlled by the FET and the constant potential of the surrounding electrode. This means that the emission current can be reduced without the need to significantly increase the source-drain voltage of the FET. Using this effect, we can control a FEA with a high electron extraction voltage using a FET with a low permitted source-drain voltage.

\section{ACKNOWLEDGMENTS}

The authors would like to thank S. Yamashita and K. Matsubara for their technical assistance. The work was supported, in part, by Shizuoka University 21st COE (Center of Excellence) Program from the Ministry of Education, Culture, Sports, Technology and Science of Japan.

${ }^{1}$ S. Yamamoto, S. Sato, and S. Fukuhara, Surf. Sci. 71, 191 (1978).

${ }^{2}$ K. Yokoo, M. Arai, M. Mori, J. Bae, and S. Ono, J. Vac. Sci. Technol. B 13, 491 (1995).

${ }^{3}$ T. Matsukawa, K. Koga, S. Kanemura, H. Tanoue, and J. Itoh, IEEE Trans. Electron Devices 46, 2261 (1999).

${ }^{4}$ M. Nagao, C. Yasumuro, Y. Sacho, H. Tanoue, S. Kanemaru, and J. Itoh, J. Vac. Sci. Technol. B 24, 936 (2006).

${ }^{5}$ M. Nagao, C. Yasumuro, M. Taniguchi, S. Itoh, S. Kanemaru, and J. Itoh, J. Vac. Sci. Technol. B 25, 464 (2007).

${ }^{6}$ H. Shimawaki, K. Tajima, H. Mimura, and K. Yokoo, IEEE Trans. Electron Devices 49, 1665 (2002). 